\title{
A therapeutic exercise program improves pain and physical dimension of health-related quality of life in young adults: a randomized controlled trial
}

\author{
Rodríguez-Romero, Beatriz PhD, MSc; Bello, Olalla PhD; Vivas Costa, Jamile \\ PhD, MSc; Carballo-Costa, Lidia PT
}

From the Psychosocial Intervention and Functional Rehabilitation Research Group, Department of Physiotherapy, Medicine and Biomedical Sciences, Faculty of Physiotherapy, University of A Coruña, A Coruña Campus, A Coruña, Spain (BR-R, JVC, LC-C); and Learning and Control of Human Movement Research Group, Department of Physiotherapy, Medicine and Biomedical Sciences, Faculty of Physiotherapy, University of A Coruña, A Coruña Campus, A Coruña, Spain (OB).

\begin{abstract}
Objective. The aim of this study was to evaluate the effects of an exercise therapy program on pain and physical dimension of health-related quality of life for young adults with musculoskeletal pain.

Design. This is a randomized controlled single-blind trial. Fifty-seven subjects (58\% women) were randomly assigned to experimental [ $n=28,21.4$ (2.9) yrs] and control [ $\mathbf{n}=29,21.0$ (4.2) yrs] groups. The experimental group participated in a 9-wk stabilization exercise therapy program, $60 \mathrm{mins} / \mathrm{wk}$, whereas the control group did not exercise, with a preintervention and postintervention assessment. Primary outcome was Physical Component Summary of SF-36. Secondary outcomes were Nordic Musculoskeletal Questionnaire, Visual Analogue Scale, Oswestry Disability Index, Neck Disability Index, and Trunk Flexor Endurance Test. The Shapiro-Wilk, independent $\mathrm{t}$ test or Mann-Whitney $U$ test, $\mathrm{X}^{2}$, or Fisher's exact test were used for statistical analysis.

Results. After intervention, the experimental group improved by 3.2 (4.5) points on the Physical Component Summary $(P=0.01)$, decreased prevalence of low back pain in the last month $(P=0.02)$ and cervical disability $(P=0.02)$, and increased flexor trunk endurance $(P=0.005)$.

Conclusions. This study confirmed that a 9-wk progressive exercise therapy program can improve physical health and reduce the prevalence of cervical disability and low back pain in the last month in young adults with musculoskeletal pain.
\end{abstract}


Musculoskeletal pain (MSP) is a significant health problem in society and has been shown to have a major impact on health-related quality of life, for both physical and mental health (MH), ${ }^{1-3}$ and this may be even more relevant in a young population. Most people who experience this type of pain go on to have recurrent episodes. ${ }^{3}$ The prevalence of MSP in younger people, such as university students, has increased significantly in the last decade because of different factors such as sedentary lifestyle with increased use of computers, low physical activity levels, poor sleeping habits, or psychosocial factors. ${ }^{4}$ The most common locations with symptoms include neck and shoulder regions, lumbar region, and simultaneously in multiple other regions. ${ }^{4}$ They may have a number of negative effects, including more generalized and chronic pain. ${ }^{1,2}$ Considering the increasingly higher prevalence of MSP in young adults and the predictive value that this pain has on suffering MSP as an adult, it seems appropriate to focus greater attention on early prevention at a young age.

Exercise therapy has been investigated extensively, and there is evidence that it is effective for the prevention and treatment of low back and cervical pain. ${ }^{5,6}$ Although there is limited evidence regarding the specific content of exercises, their intensity, as well as the number, duration, and frequency of sessions; the accumulated evidence ${ }^{5-9}$ on the topic provides the best indications for which and how therapeutic exercises should be used. In the case of subjects with lower back pain (LBP), the most recommended exercises consist of coordination, relaxation, and resistance of the trunk muscles, ${ }^{7}$ as well as motor control or dynamic stabilization exercises and integrated activation of the global muscles. ${ }^{9}$ In turn, in subjects with neck pain, the recommended methods involve strengthening, resistance, stretching, and stabilization exercises. ${ }^{6,8}$

Despite the available evidence regarding the effects of exercise therapy on the prevention of MSP, we have found hardly any studies that apply this to young adults. ${ }^{10}$ However, we consider that more attention should be paid to this collective because of their imminent incorporation in the labor market, and the socio-economic consequences these symptoms may have for these future workers and their employers.

Taking into consideration the increase in MSP in younger persons, the evidence supporting exercise therapy for its prevention in adults, and at the same time the lack of consensus regarding the specific characteristics of the intervention program, this study aims to evaluate the effect of a progressive, multimodal therapeutic exercise program on pain, physical health, disability, and trunk flexor muscle endurance in a group of young university adults.

\section{METHODS}

This study was reviewed and approved by the ethics committee of the University of A Coruña (Approval Number and Data: CE 19/2016, September 8, 2016) and strictly followed the procedures in accordance with the Helsinki Declaration. This was a 9-wk, single-blind, parallelgroup, randomized controlled study with two measurement points (baseline and 3 mos). This study conforms to all CONSORT guidelines and reports the required information accordingly (see Supplementary Checklist, Supplemental Digital Content 1, http://inks.lww.com/PHM/A711). Detailed information about the study was provided to all the participants before their enrollment, although they were blinded about the study hypothesis. A written informed consent was obtained from them. In addition, the study was registered in Protocol Registration and Results System with Identifier Number NCT03529864.

The present study was conducted on university students of the Physiotherapy's Bachelor Degree at University of A Coruña (Spain). Subjects were randomly assigned to either the exercise or the control group using a list of randomized number obtained of http://www.randomization.com/ by a second researcher who did not take part either in the evaluation or in the intervention. The main researcher is a physiotherapist (with a PhD), whose expertise in the area of musculoskeletal pain spans more than 20 yrs. The experimental group 
participated in the progressive exercise therapy program. In contrast, the control group did not receive any type of information or instructions apart from the general information sheet on the progress of the study, attached to the informed consent form.

Participants with any of the following conditions were excluded from the study: having any type of cardiovascular, neuromusculoskeletal, or systemic diseases that restricted exercise participation, not accepting to take part in the study or not attending the initial evaluation sessions. The inclusion criteria consisted of second-year university students of degree in physiotherapy, who gave their informed consent to participate. They could report musculoskeletal pain in any area of the body.

\section{Outcome Measurement}

\section{Primary Outcome Measure}

This was health-related quality of life, measured by the Physical Component Summary (PCS) of the SF-36questionnaire (version 2). ${ }^{11}$ It assesses the state of health in the following eight dimensions: physical function (PF), role physical (RP), bodily pain (BP), general health (GH), vitality (VT), social function, role-emotional, and $\mathrm{MH}$. These dimensions may be reduced to two scores: PCS and Mental Component Summary. To interpret the results, standardized scores are obtained with the values for the reference population standards, whereby a score of $50(\mathrm{SD}=10)$ represented the mean for the general population, and a score of 10 was one SD. Values of 50 or less have to be respectively interpreted as better or worse than those of the reference population. The psychometric characteristics of the SF-36 have been studied extensively and have been found to be reliable, valid, and sensitive. ${ }^{11}$ Minimally important difference for SF-36 seems to be approximately half an SD (5 points). ${ }^{12}$

\section{Secondary Outcome Measure}

These were MSP prevalence, pain intensity, lumbar and cervical disability, and trunk flexor muscle endurance.Prevalence and consequences of MSP were measured using the Nordic Musculoskeletal Questionnaire (NMQ), ${ }^{13}$ which consists of a rear view of the human body, divided into nine anatomical regions. The test-retest reliability and the reproducibility of this questionnaire have been demonstrated. ${ }^{13}$

The MSP intensity in these nine regions (neck, shoulders, upper back, elbows, wrists/hands, lower back, hips/thighs, knees, ankles/feet), for the previous 4 wks, was measured with the Visual Analog Scale (VAS). ${ }^{14}$ There is much evidence supporting the validity of VAS. ${ }^{14}$ The recommended cutoff points were used to interpret the scores: no pain (0-4 mm), slight pain (5-44 $\mathrm{mm})$, moderate pain (45-74 $\mathrm{mm})$, and severe pain (75-100 mm).

Disability by lumbar pain was measured with Oswestry Disability Index. ${ }^{15}$ This is one of the most widely used and recommended scales, and is viable and easy to administer and score, and has suitable measurement features. ${ }^{15}$ The total score of this index is expressed as a percentage. Between $0 \%$ and $20 \%$ is minimal lumbar disability; from $21 \%$ to $40 \%$, moderate disability; from $41 \%$ to $60 \%$, severe disability; from $61 \%$ to $80 \%$, crippled; and from $81 \%$ to $100 \%$, bed-bound or exaggerating their symptoms. Four points are considered as the minimum difference for clinically significant change. ${ }^{15}$

In addition, disability by cervical pain was measured with Neck Disability Index. ${ }^{16}$ The total score is out of 50 points: from 0 to 4 points, no disability; from 5 to 14 points, mild disability; from 15 to 24 points, moderate disability; from 25 to 34 points, severe disability; and from 35 to 50 points, complete disability. A minimum clinically important difference value of 3 to 5 points can be confidently applied in a practice setting. 
For evaluating flexor trunk endurance, the test validated by McGill et al. ${ }^{17}$ [with normative data of 147 (90) secs] was performed.

Perception of changes after the treatment and a measurement of satisfaction, carried out using a seven-point Likert-type scale, were only measured after the intervention. The scale used ranges from "completely improved" to "worse than ever" measure perception of the changes and from "extremely satisfied" to "extremely unsatisfied" to measure satisfaction. ${ }^{18}$

The participants also provided information about demographic, academic, employment, and lifestyle characteristics using a self-administered questionnaire and about patterns of physical activity using the International Physical Activity Questionnaire (IPAQ) and Charlson Comorbidity Index.

\section{Intervention}

The experimental group took part in a progressive exercise therapy program. Table 1 describes the intervention according to the TIDieR checklist (why, what, who provided, how, where, when and how much, tailoring, modifications, and how well about the intervention). The exercise therapy program consisted of group sessions with eight or nine participants, with each session lasting 60 mins, supervised by the principal researcher, for 9 consecutive weeks once a week. Exercises involving body scanner and breathing pattern re-education; neutral lumbar-pelvic position control in all directions; transverse abdominis activation; neutral cervical position control; global stabilizer activation exercises for the lumbar-pelvic and cervical-scapular regions; lower back flexibility; and muscular strengthening and stretching were included. 


\begin{tabular}{|c|c|}
\hline Brief name & Progressive exercise therapy program. \\
\hline Why & $\begin{array}{l}\text { The design of the intervention took into account the available evidence for the prevention and } \\
\text { treatment of neck and low back pain. }\end{array}$ \\
\hline \multirow[t]{2}{*}{ What } & $\begin{array}{l}\text { Materials: mats, fit-balls, TheraBands, towels, Dyn-Air cushions, chairs, stretchers. Procedures: } 3 \\
\text { levels of exercises of increasing difficulty. }\end{array}$ \\
\hline & $\begin{array}{l}\text { Standing warm-up exercises. Exercises involving: body scanner and breathing pattern re-education, } \\
\text { neutral lumbar-pelvic position control in all directions; transverse abdominis activation; neutral } \\
\text { cervical position control; global stabilizer activation exercises for the lumbar-pelvic and cervical- } \\
\text { scapular regions; low back flexibility; and muscular strengthening and stretching. Some examples } \\
\text { of these exercises were: abdominal draw-in maneuver in isolation, in different positions and during } \\
\text { progressive exercises (heel slides, leg lifts, dead bug exercise, etc.); bridging (side bridge with } \\
\text { knees flexed and extended, supine and prone bridge); quadruped with alternate arm/leg; lunge; } \\
\text { squat; cervical-scapular stabilization exercise (head nods, cervical bracing, static and dynamic } \\
\text { exercises to appropriate scapular control position). }\end{array}$ \\
\hline Who provided & Physiotherapist. \\
\hline How & Face to face, group with 8-9 subjects. \\
\hline Where & Laboratory. \\
\hline \multirow{4}{*}{$\begin{array}{l}\text { When and how } \\
\text { much }\end{array}$} & From October to December 2014. \\
\hline & 9 consecutive weeks, once a week. \\
\hline & Each session: 60 mins. \\
\hline & $\begin{array}{l}\text { Progress consideration: whether the subject was capable of remaining in a neutral spine position } \\
\text { and not change position, together with the patient's perception of the difficulty involved. The } \\
\text { progression of the exercises consisted of making the starting position more difficult, increasing the } \\
\text { number of repeats and contraction time, and increasing the load (with external resistance and body } \\
\text { weight) through more dynamic and functional exercises. }\end{array}$ \\
\hline Tailoring & $\begin{array}{l}\text { Techniques to facilitate learning were used, such as individual exteroceptive palpation and verbal } \\
\text { commands for the whole group. }\end{array}$ \\
\hline Modifications & $\begin{array}{l}\text { If subjects felt pain during any of the exercises, they were told to find a position in which they no } \\
\text { longer felt any pain, or otherwise they were told to stop the exercise. }\end{array}$ \\
\hline \multirow[t]{2}{*}{ How well } & $\begin{array}{l}\text { The subjects' attendance was registered, and they were encouraged to take part in each session by } \\
\text { the physiotherapist. }\end{array}$ \\
\hline & $\begin{array}{l}\text { The intervention was delivered as planned and evaluated from the perspective of the participant, } \\
\text { based on their degree of satisfaction and perception of changes. }\end{array}$ \\
\hline
\end{tabular}

\section{Sample Size Calculation}

According to Norman et al., ${ }^{12}$ the primary endpoint with respect to the efficacy of exercise therapy program was an improvement of 4 points with an SD of 5 points on the PCS of SF-36, from baseline to 9 wks of intervention. To detect an increase in PCS of 4 points $(S D=5)$, with a two-sided 5\% significance level and a power of $80 \%$, a sample size of 29 patients per group was necessary, given an anticipated dropout rate of $15 \%$.

\section{Statistical Analysis}

Statistical analysis was performed using Statistical Package for Social Sciences for Windows Version 19.0 (SPSS Inc, Chicago, IL), with statistical significance set at $\mathrm{P}<0.05$. The continuous data were assumed as the mean (SD), and the categorical data were assumed as the number and percentage. The comparison of subjects was carried out in two stages: (a) at the start of the study, to conform the homogeneity of the groups, and (b) after the intervention, as differences between initial and final evaluation, to detect any statistically significant changes in the variables of interest. The Shapiro-Wilk test was used to contrast the normal distribution of the variables. The independent $t$ test or Mann-Whitney $\mathrm{U}$ test was used to compare continuous data, and X2 or Fisher's exact test was calculated for categorical and proportional data. 


\section{RESULTS}

Figure 1 shows the CONSORT flow diagram demonstrating the participants' progress throughout the study. The study concluded with 57 participants (81.4\%): 28 subjects in the exercise group and 29 in the control group. Two subjects stopped the treatment, one for generalized pain, and another because of incompatibilities with the timetable. In the exercise group, $86 \%$ attended eight or nine sessions. The groups were similar for practically all of the variables at the start of the study.

Enrollment

Eligibility $(n=70)$

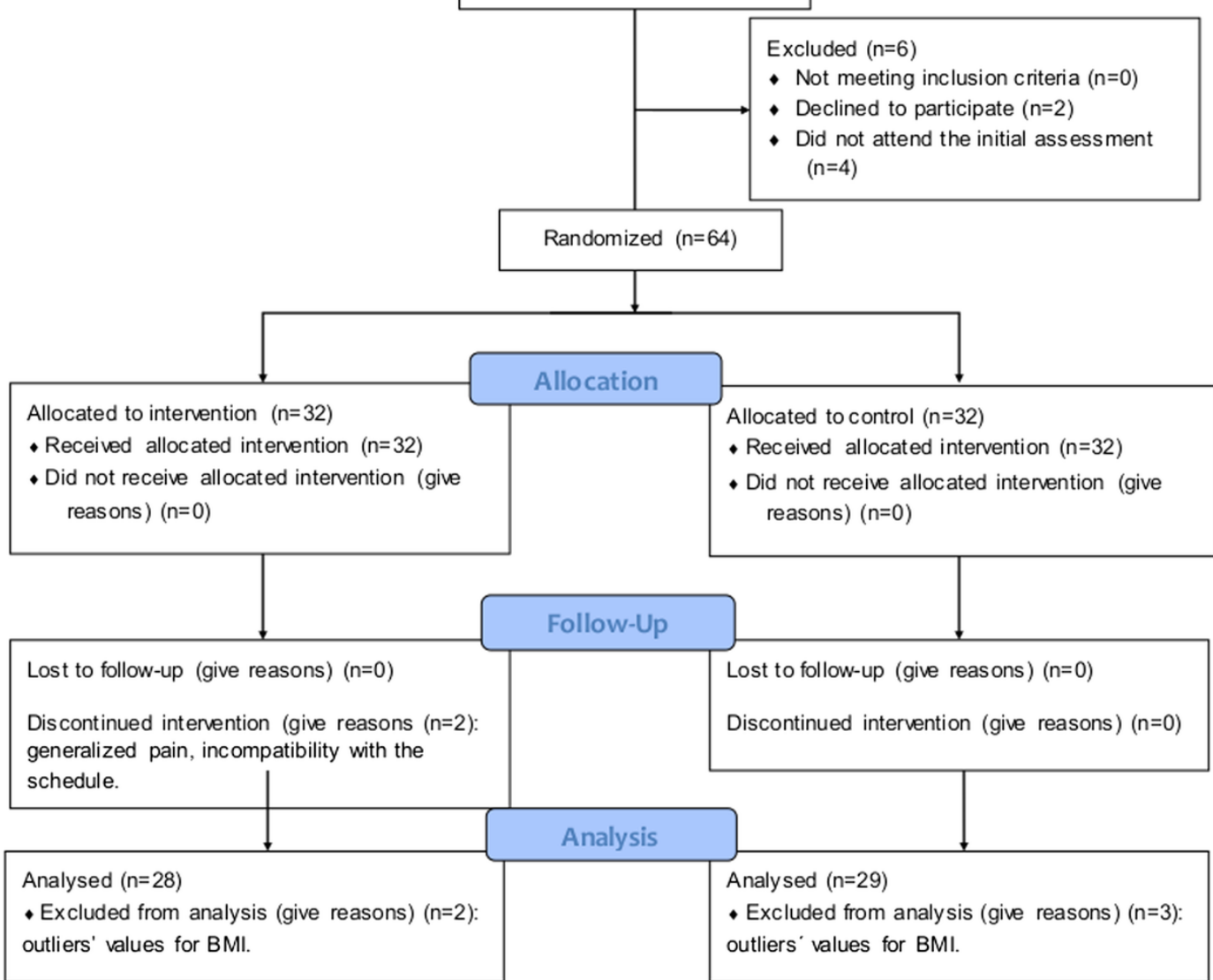

FIGURE 1. Flow diagram showing the participants' progress throughout the different phases of the study. 
The anthropometric data, sociodemographic and lifestyle characteristics, standardized scores from the SF-36, the results on the prevalence and intensity of MSP, information on lumbar and neck disability, and flexor trunk endurance for both groups, are shown in Table 2. The participants were young subjects and mainly female. In both groups, the body mass index values were within the normal interval, with a level of physical activity classified as "active" (physical activity that benefits health); the majority were nonsmokers, and they did not have any comorbidities. The subjects had a slightly higher score than the population reference values [50 (10)] in most dimensions of SF-36, although they did have lower values in the dimensions of BP [45.5 (6.1)] and VT [48.4 (6.4)]. In general, the locations with a higher prevalence of MSP were the neck, upper and lower back, and the shoulders. Thus, only the results for these four regions are included in the article. Furthermore, only the results relating to the most relevant questions from the NMQ are shown. As a whole, the subjects had a slight pain intensity (VAS scores between 0.5 and 4.4) in the different body areas. Both groups had minimum functional limitation for the low back (Oswestry Disability Index) and neck (Neck Disability Index). The values for the flexor trunk endurance test were lower than the reference values of McGill et al. ${ }^{17}$ [147 (90)], although they were more homogeneous, because the SD was much lower. 
TABLE 2 Participants' baseline characteristics

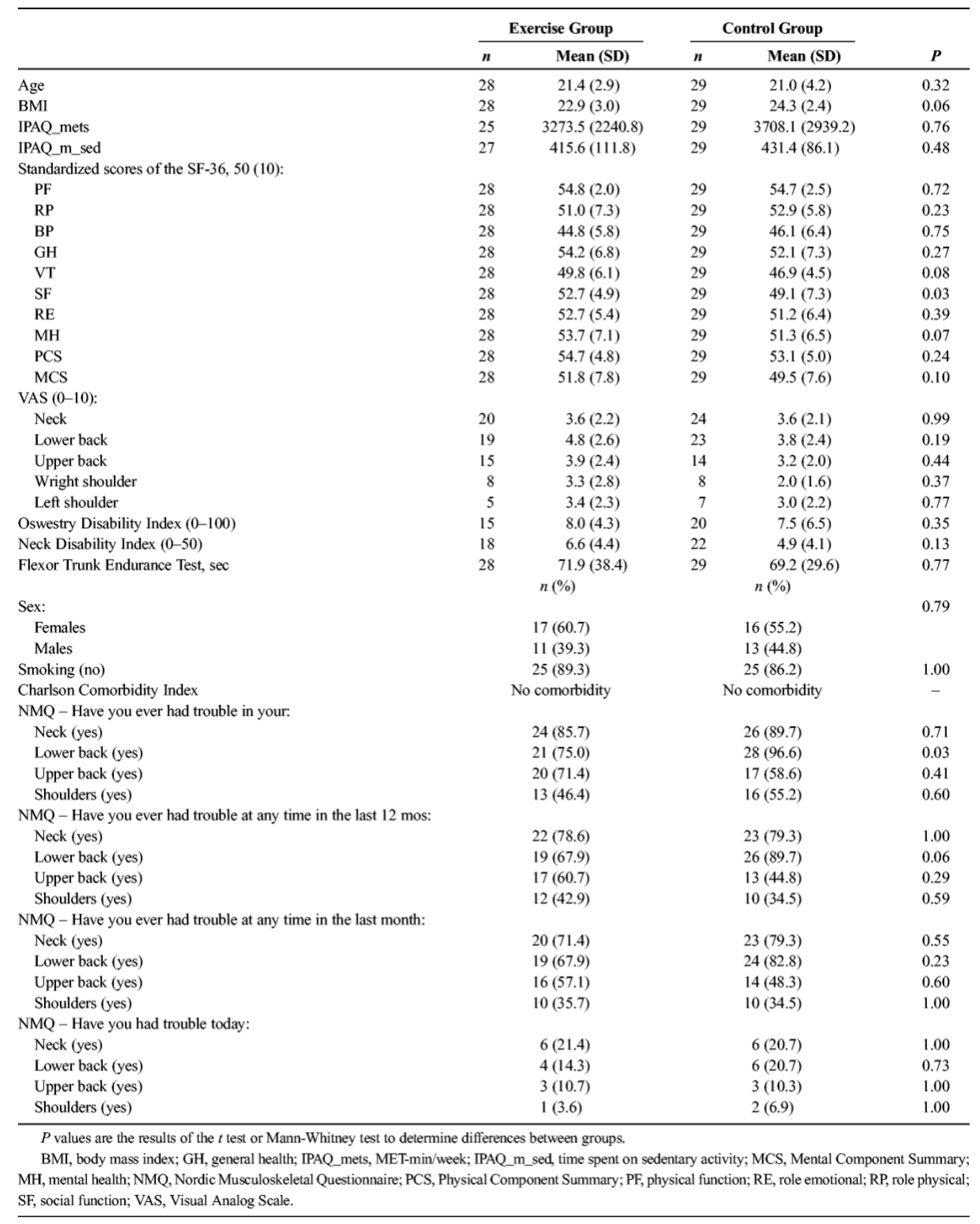


After the intervention, the physical health was significantly improved in the exercise group (PCS, $\mathrm{P}=0.01$ ) in comparison with the control group. There was a difference of 3.2 (4.5) points in the exercise group and 0.3 (3.9) points in the control group (Table 3). In the four dimensions that most contribute to the PCS score (physical function, role physical, BP, and general health), there was an improvement with respect to the control group, although this was only significant for the $\mathrm{BP}(\mathrm{P}=0.03)$. The results revealed that the exercise group had an improvement in physical health component, demonstrating that the intervention had a significant influence.

TABLE 3 Differences between the final evaluation and initial evaluation in terms of primary and secondary outcomes in the exercise and control groups

\begin{tabular}{|c|c|c|c|c|c|}
\hline & \multicolumn{2}{|c|}{ Exercise Group } & \multicolumn{2}{|c|}{ Control Group } & \multirow[b]{2}{*}{$P$} \\
\hline & $n$ & Mean (SD) & $n$ & Mean (SD) & \\
\hline \multicolumn{6}{|l|}{ Primary outcomes } \\
\hline \multicolumn{6}{|l|}{ SF-36 dimensions: } \\
\hline PF & 28 & $0.3(2.2)$ & 29 & $-0.5(1.6)$ & 0.25 \\
\hline $\mathrm{RP}$ & 28 & $3.3(5.5)$ & 29 & $1.0(4.3)$ & 0.11 \\
\hline $\mathrm{BP}$ & 28 & $4.0(7.2)$ & 29 & $0.2(5.4)$ & 0.03 \\
\hline $\mathrm{GH}$ & 28 & $0.4(3.7)$ & 29 & $-0.7(5.4)$ & 0.29 \\
\hline PCS & 28 & $3.2(4.5)$ & 29 & $0.3(3.9)$ & 0.01 \\
\hline \multicolumn{6}{|l|}{ Secondary outcomes } \\
\hline \multicolumn{6}{|l|}{ VAS $(0-10)$ : } \\
\hline Neck & 14 & $0.4(2.0)$ & 20 & $0.0(2.1)$ & 0.54 \\
\hline Lower back & 11 & $-1.4(2.9)$ & 16 & $0.3(1.9)$ & 0.09 \\
\hline Upper back & 7 & $0.1(3.2)$ & 11 & $-0.1(2.2)$ & 0.89 \\
\hline Wright shoulder & 3 & $0.8(1.6)$ & 4 & $2.4(1.0)$ & 0.16 \\
\hline Left shoulder & 3 & $1.0(0.9)$ & 5 & $0.1(1.7)$ & 0.40 \\
\hline Oswestry Disability Index $(0-100)$ & 12 & $-2.5(4.2)$ & 19 & $-1.3(6.9)$ & 0.58 \\
\hline Neck Disability Index $(0-50)$ & 17 & $-2.7(3.8)$ & 24 & $0.2(2.4)$ & 0.02 \\
\hline \multirow[t]{2}{*}{ Flexor Trunk Endurance Test, sec } & 28 & $112.3(58.9)$ & 29 & $63.9(59.3)$ & 0.005 \\
\hline & & $n(\%)$ & & $n(\%)$ & \\
\hline \multicolumn{6}{|c|}{ NMQ - Have you ever had trouble at any time in the last month: } \\
\hline Neck (yes) & & $15(53.6)$ & & $21(72.4)$ & 0.14 \\
\hline Lower back (yes) & & $12(42.9)$ & & $21(72.4)$ & 0.02 \\
\hline Upper back (yes) & & $9(32.1)$ & & $14(48.3)$ & 0.22 \\
\hline Shoulders (yes) & & $8(28.6)$ & & $8(27.6)$ & 0.93 \\
\hline \multicolumn{6}{|l|}{ NMQ - Have you had trouble today: } \\
\hline Neck (yes) & & $3(10.7)$ & & $8(27.6)$ & 0.11 \\
\hline Lower back (yes) & & $3(10.7)$ & & $4(13.8)$ & 0.99 \\
\hline Upper back (yes) & & $0(0.0)$ & & $2(6.9)$ & 0.49 \\
\hline Shoulders (yes) & & $0(0.0)$ & & $2(6.9)$ & 0.49 \\
\hline
\end{tabular}

$P$ indicates the result of the $t$ test or Mann-Whitney test to determine differences between groups.

GH, general health; NMQ, Nordic Musculoskeletal Questionnaire; PCS, Physical Component Summary; PF, physical function; RP, role physical; VAS, Visual Analog Scale.

In turn, the intervention led to statistically significant improvements in the secondary outcomes (Table 3), such as in cervical disability (Neck Disability Index) $(\mathrm{P}=0.02)$, which was reduced by a mean (SD) of 2.7 (3.8) points in the exercise group compared with the control group, and in the flexor trunk muscle endurance ( $\mathrm{P}=0.005)$, which improved by a mean (SD) of 112.3 (58.9) points in this group in comparison with control group. In the NMQ on the prevalence of MSP (Table 3), a significant reduction $(\mathrm{P}=0.02)$ was also obtained in the prevalence of LBP during the last month in the exercise group. In this group, it was referred to by $42.9 \%$ of the subjects, compared with $72.4 \%$ in the control group. After the intervention, most subjects were very satisfied (67.9\%) with the results and slightly improved (60.7\%). 


\section{DISCUSSION}

The main aim of this study was to determine whether a therapeutic exercise program, based on stabilization, improved the physical health of young adults and, as secondary aims, to determine its effect on the prevalence of MSP in different body regions, on the intensity of the MSP, on the disability produced by it, and on trunk flexor muscle endurance.

This study confirmed that the proposed program can effectively improve physical component health (PCS) and flexor trunk muscle endurance, as well as reducing the prevalence of LBP in the last month, and disability due to neck pain in university students.

The improvement in physical component health (PCS) [mean (SD) = 3.2 (4.5)] could mainly be due to the improvement obtained in the dimension BP [4.0 (7.2) points], which means that the subjects in the exercise group had less pain and less impact because of pain in their daily life, after the intervention. The two measures, PCS and BP, were shown to have a good sensitivity to this change. ${ }^{19}$

The SF-36 is a recommended result measure in studies on $\mathrm{MSP}^{19}$ and an increasing number have demonstrated the effects of exercise therapy on health. ${ }^{20-23}$ Our findings are in line with these previous studies, although these are very heterogeneous and differ from ours in terms of the type of participants (older patients with chronic pain). Cuesta et al. ${ }^{20,21}$ found improvements in the PCS of 1.74 points $^{20}$ and of between 8.9 and 10.6 points $^{21}$ in patients with LBP and 0.65 in patients with neck pain. ${ }^{20}$ Moussouli et al., ${ }^{22}$ who studied the efficacy of stabilization exercises in LBP, demonstrated a significant improvement in the BP and VT dimensions of SF-36, which remained stable after 9 mos. In turn, Celenay et al., ${ }^{23}$ who studied patients with neck pain, found an improvement of 2.9 points on the PCS.

In the case of the secondary result measures, the descriptive data reveal that the prevalence of MSP, recorded at different periods (ever last year, last month, and today) is very high in this group of young subjects, although its intensity was slight. Neck pain and LBP were the most frequent (78.9\% for neck pain and LBP, for all group, 1-yr prevalence), and the prevalence ratios are within the ranges reported for the adult population (1-yr prevalence ranges from $0.8 \%$ to $82.5 \%$ for LBP and from $4.8 \%$ to $79.5 \%$ for neck pain). ${ }^{1,2}$ They are also consistent with those from other studies carried out with university students in the field of health sciences, ${ }^{24-26}$ although our sample presented slightly higher values.

Despite the high prevalence of MSP, the disability due to back and neck pain is minimum and mild, respectively. We consider that the age, pain intensity, and level of physical activity of the participants could be factors that justify these results.

In turn, the statistical analysis of the secondary result measures shows that the intervention reduced the prevalence of LBP, but not the disability it causes. We believe that this latter aspect is due to the low baseline values and that the questionnaire we used is more sensitive to changes in levels of greater disability. ${ }^{19}$ Pain decreased in the neck region, but not significantly, whereas the disability caused by this pain did decrease. Several studies that have demonstrated the effectiveness of stabilization exercises ${ }^{23,27}$ obtained greater differences than ours. This said, the subjects in those studies had higher baseline values, greater pain intensity, and were older, among other differences. In any event, our results are in line with those of other authors, indicating that stabilization exercises reduce neck disability. 
The intervention also proved to be effective in terms of increasing the resistance of the abdominal muscles. The baseline values were lower than the reference values ${ }^{17}$ for the entire sample and improved after the intervention in the exercise group, exceeding these reference values. Although it would seem that one single test is not enough to measure the active spinal stabilization system, the test we used has proved to be highly reliable for this purpose. ${ }^{28}$ Although there are very few studies that use this variable, Shamsi et al. ${ }^{29}$ also found significant improvements after using stabilization exercises.

With regard to the changes perceived, considered as a sensitive, valid measurement of the benefits of the intervention, ${ }^{30}$ a total of $21 \%$ stated that they felt much better, and the majority (61\%) stated that they felt slightly better. We believe that these results could be due to the low intensity and disability at the baseline, as well as the improvement in the BP dimension and in muscular resistance.

This study does have some limitations. Study subjects were only recruited from the Faculty of Physiotherapy, meaning that they do not reflect the entire spectrum of university students, and thus, the results cannot be generalized to the population as a whole. Furthermore, the sustained benefits of the intervention are unknown, because no long-term follow-up was carried out.

\section{CONCLUSIONS}

This study confirmed that the proposed progressive exercise therapy program, carried out once a week for 9 consecutive weeks, can effectively improve Physical component health (PCS) and flexor trunk muscle endurance, as well as reducing the prevalence of LBP in the last month, and disability caused by neck pain, in young university adults with musculoskeletal pain. The proposed exercise program may be recommended to young university adult with neck and LBP, at least in the short term.

\section{ACKNOWLEDGMENTS}

We thank all of the students from the Faculty of Physiotherapy who took part in the study. We also thank the management team for allowing us to use the facilities and Lorissa Achjian for checking and correcting the English in the article.

\section{REFERENCES}

1. Hoy D, Brooks P, Blyth F, et al: The epidemiology of low back pain. Best Pract Res Clin Rheumatol 2010;24:769-81

2. Hoy DG, Protani M, De R, et al: The epidemiology of neck pain. Best Pract Res Clin Rheumatol 2010;24:783-92

3. Salaffi F, De Angelis R, Stancati A, et al: Health-related quality of life in multiple musculoskeletal conditions: a cross-sectional population based epidemiological study. II. The MAPPING study. Clin Exp Rheumatol 2005;23:829-39

4. Oksanen AM, Laimi K, Löyttyniemi E, et al: Trends of weekly musculoskeletal pain from 2000 to 2012: national study of Finnish university students. Eur J Pain 2014;18:1316-22

5. Steffens D, Maher CG, Pereira LS, et al: Prevention of low back pain: a systematic review and meta-analysis. JAMA Intern Med 2016;176:199-208

6. Bertozzi L, Gardenghi I, Turoni F, et al: Effect of therapeutic exercise on pain and disability in the management of chronic nonspecific neck pain: systematic review and meta-analysis of randomized trials. Phys Ther 2013;93:1026-36

7. Delitto A, George SZ, Van Dillen LR, et al: Low back pain. J Orthop Sports Phys Ther 2012;42:A1-57 
8. Childs JD, Cleland JA, Elliott JM, et al: Neck pain: clinical practice guidelines linked to the international classification of functioning, disability, and health from the orthopedic section of the American Physical Therapy Association. J Orthop Sports Phys Ther 2008;38:A1-34

9. Byström MG, Rasmussen-Barr E, Grooten WJ: Motor control exercises reduces pain and disability in chronic and recurrent low back pain: a meta-analysis. Spine (Phila Pa 1976) 2013;38:E350-8

10. Kim D, Cho M, Park Y, et al: Effect of an exercise program for posture correction on musculoskeletal pain. J Phys Ther Sci 2015;27:1791-4

11. Vilagut G, Valderas JM, Ferrer M, et al: Interpretation of SF-36 and SF-12 questionnaires in Spain: physical and mental components [in Spanish]. Med Clin (Barc) 2008;130:726-35

12. Norman GR, Sloan JA, Wyrwich KW: Interpretation of changes in health-related quality of life: the remarkable universality of half a standard deviation. Med Care 2003;41:582-92

13. Dawson AP, Steele EJ, Hodges PW, et al: Development and test-retest reliability of an extended version of the Nordic Musculoskeletal Questionnaire (NMQ-E): a screening instrument for musculoskeletal pain. J Pain 2009;10:517-26

14. Hawker GA, Mian S, Kendzerska T, et al: Measures of adult pain: Visual Analog Scale for Pain (VAS Pain), Numeric Rating Scale for Pain (NRS Pain), McGill Pain Questionnaire (MPQ), Short-Form McGill Pain Questionnaire (SF-MPQ), Chronic Pain Grade Scale (CPGS), Short Form-36 Bodily Pain Scale (SF-36 BPS), and Measure of Intermittent and Constant Osteoarthritis Pain (ICOAP). Arthritis Care Res (Hoboken) 2011;63(Suppl 11):S240-52

15. Fairbank JC, Pynsent PB: The Oswestry Disability Index. Spine (Phila Pa 1976) 2000;25:2940-52; discussion 52

16. Andrade Ortega JA, Delgado Martínez AD, Almécija Ruiz R: Validation of the Spanish version of the Neck Disability Index. Spine (Phila Pa 1976) 2010;35:E114-8

17. McGill SM, Childs A, Liebenson C: Endurance times for low back stabilization exercises: clinical targets for testing and training from a normal database. Arch Phys Med Rehabil 1999;80:941-4

18. Dworkin RH, Turk DC, Farrar JT, et al: Core outcome measures for chronic pain clinical trials: IMMPACT recommendations. Pain 2005;113:9-19

19. Walsh TL, Hanscom B, Lurie JD, et al: Is a condition-specific instrument for patients with low back pain/leg symptoms really necessary? The responsiveness of the Oswestry Disability Index, MODEMS, and the SF-36. Spine (Phila Pa 1976) 2003;28:607-15

20. Cuesta-Vargas AI, González-Sánchez M, Casuso-Holgado MJ: Effect on health-related quality of life of a multimodal physiotherapy program in patients with chronic musculoskeletal disorders. Health Qual Life Outcomes 2013;11:19

21. Cuesta-Vargas AI, García-Romero JC, Arroyo-Morales M, et al: Exercise, manual therapy, and education with or without high-intensity deep-water running for nonspecific chronic low back pain: a pragmatic randomized controlled trial. Am J Phys Med Rehabil 2011;90:526-34; quiz 358

22. Moussouli M, Vlachopoulos SP, Kofotolis ND, et al: Effects of stabilization exercises on health-related quality of life in women with chronic low back pain. J Phys Act Health 2014;11:1295-303

23. Celenay ST, Akbayrak T, Kaya DO: A comparison of the effects of stabilization exercises plus manual therapy to those of stabilization exercises alone in patients with nonspecific mechanical neck pain: a randomized clinical trial. J Orthop Sports Phys Ther 2016;46:44-55

24. Nyland LJ, Grimmer KA: Is undergraduate physiotherapy study a risk factor for low back pain? A prevalence study of LBP in physiotherapy students. BMC Musculoskelet Disord 2003;4:22

25. Falavigna A, Teles AR, Mazzocchin T, et al: Increased prevalence of low back pain among physiotherapy students compared to medical students. Eur Spine J 2011;20:500-5

26. Leggat PA, Smith DR, Clark MJ: Prevalence and correlates of low back pain among occupational therapy students in Northern Queensland. Can J Occup Ther 2008;75:35-41

27. Dusunceli Y, Ozturk C, Atamaz F, et al: Efficacy of neck stabilization exercises for neck pain: a randomized controlled study. J Rehabil Med 2009;41:626-31

28. Ozcan Kahraman B, Salik Sengul Y, Kahraman T, et al: Developing a reliable core stability assessment battery for patients with nonspecific low back pain. Spine (Phila Pa 1976) 2016;41:E844-50

29. Shamsi MB, Rezaei M, Zamanlou M, et al: Does core stability exercise improve lumbopelvic stability (through endurance tests) more than general exercise in chronic low back pain? A quasirandomized controlled trial. Physiother Theory Pract 2016;32:171-8

30. Dworkin RH, Turk DC, Wyrwich KW, et al: Interpreting the clinical importance of treatment outcomes in chronic pain clinical trials: IMMPACT recommendations. J Pain 2008;9:105-21 
